\title{
Action Potential Bursts Modulate the NMDA-R Mediated Spike Timing Dependent Plasticity in a Biophysical Model
}

\author{
Vassilis Cutsuridis \\ Center for Memory and Brain, Boston University, Boston, MA, USA \\ vcut@bu.edu
}

\begin{abstract}
Spike timing dependent plasticity (STDP) requires the temporal association of presynaptic and postsynaptic action potentials (APs). However, some synapses in the CA1 region of the hippocampus are suprisingle unreliable at signaling the arrival of single spikes to the postsynaptic neuron [4. In such unreliable synapses pairing of excitatory postsynaptic potentials (EPSPs) and single APs at low frequencies is ineffective at generating plasticity [2, 3]. A recent computational study [7. has shown that the shape of the STDP curve strongly depends on the burst interspike interval in the presence/absence of inhibition when a presynaptic dendritic burst and a postsynaptic somatic spike were paired together. In this study, we investigate via computer simulations the conditions under which STDP is affected when now a high frequency somatic burst instead of a single spike is paired with another dendritic spike. We show that during such pairing conditions in the absence of inhibition a symmetric STDP profile with a distinct positive LTP region is evident at 10-30ms interstimulus interval and flat LTD tails at all other interstimulus intervals. The symmetry is preserved at all burst interspike intervals. When inhibition is present, the STDP profile shape into a Mexican hat shaped one or an inverted symmetrical one with flat LTP tails.
\end{abstract}

Keywords. Computer model, bursts, STDP, GABA inhibition, LTP, LTD, calcium.

\section{Introduction}

Experimental studies in pyramidal neurons in the hippocampus have shown that the magnitude and sign of changes in synaptic strength depend critically on the precise timing of pre- and postsynaptic action potentials (APs), with postsynaptic APs preceding EPSPs typically leading to long-term depression (LTD), while APs evoked just after EPSP onset typically lead to long-term potentiation (LTP) [5]. Spike-timing-dependent plasticity (STDP) is an extension of Hebb's law, which states that neurons that simultaneously fire together, they wire together [1. While the cellular mechanisms underlying STDP are not well understood, most studies agree NMDA receptors play an important role because they are able to detect coincident pre- and postsynaptic activity via relief of voltage-dependent magnesium block [5]. During STDP the voltage driving the unblockage of NMDA receptors is supplied by postsynaptic APs, which actively K. Diamantaras, W. Duch, L.S. Iliadis (Eds.): ICANN 2010, Part I, LNCS 6352, pp. $107+116,2010$.
(c) Springer-Verlag Berlin Heidelberg 2010 
propagate back into the dendrites of many neuronal types . However, under certain experimental conditions single APs attenuate and can fail to backpropagate into some dendrites of pyramidal neurons [13. This failure of AP backpropagation can be rescued during high-frequency AP bursts due to boosting of AP backpropagation following AP summation and the generation of dendritic calcium spikes [3. Consistent with the potential role of AP burst-evoked dendritic calcium spikes in synaptic plasticity, a number of studies indicate a role of AP burst firing in STDP [3]. These findings prompted me to investigate how STDP is affected when a somatic burst is paired with a dendritic spike in the presence of a high frequency inhibition (GABA). Previous work from my group [6], 7], 8] has shown that the experimentally observed asymmetric STDP curve in the hippocampus undergoes an asymmetry-to-symmetry transition [10, 11], [12, which depends on the frequency of inhibition (theta vs. gamma), the conductance value of GABA inhibition, the relative timing between the GABAergic spike train and the excitatory pre-postsynaptic interstimulus interval, and on the burst interspike interval.

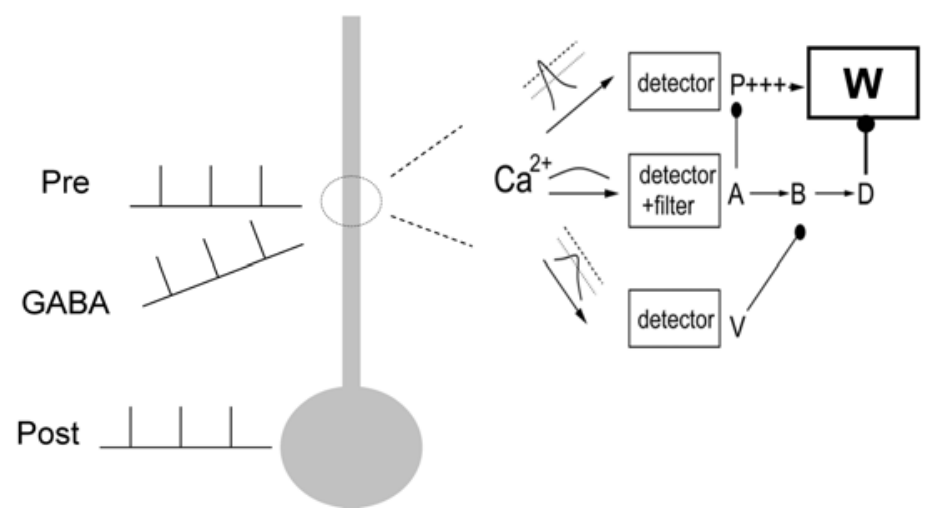

Fig. 1. Model CA1 neuron with its three transient inputs to the soma and dendrite. Synaptic plasticity at the dendritic synapses (circled region) is governed by a model calcium detector system [9. P detector: potentiation detector; D detector: depression detector; V detector: veto detector; W: synaptic weight.

\section{The Model}

The CA1 pyramidal cell model with its three calcium detectors used herein has been described in detail in [6], [7, 8]. Briefly, the model pyramidal cell consists of two compartments: a soma and a dendrite. The generation of action potentials is due to the interplay of a wealth of ionic currents such as $\mathrm{Na}^{+}, \mathrm{K}^{+}, \mathrm{Ca}^{2+}$ activated $\mathrm{K}^{+}$and $\mathrm{Ca}^{2+}$ currents as well as synaptic currents (AMPA, NMDA and $\mathrm{GABA}_{A}$ ). Hodgkin-Huxley mathematical formalism was used to describe the ionic and synaptic mechanisms of the modelled pyramidal cell. The model used calcium as the postsynaptic signaling agent for STDP and it has been 
shown to be consistent with classical long-term potentiation (LTP) and longterm depression (LTD) induced by several doublet stimulation paradigms in the absence and presence of inhibition [6, 7], 8].

In the model, calcium enters the neuron through: (1) voltage-gated calcium channels (VGCCs), and (2) NMDA channels located at the dendrite. VGCCs are activated by the arrival of backpropagating action potentials (BPAPs) initiated in the soma by excitatory postsynaptic spikes. The NMDA channels are activated by the synergistic action of excitatory and inhibitory postsynaptic potentials and sufficient membrane potential depolarization due to the BPAP, which removes the magnesium block and allows calcium to enter the cell. In the model, calcium influx from neither channels alone elicits plasticity. Plasticity results only from the synergistic action of the two calcium sources (NMDA and VGCC).

The mechanism for plasticity has a modular structure consisting of three biochemical detectors, which respond to the instantaneous calcium level and its time course in the dendrite. The detection system consists of: (1) a potentiation $(\mathrm{P})$ detector which detects calcium levels above a high-threshold $(4 \mu \mathrm{M})$ and triggers LTP, (2) a depression (D) detector which detects calcium levels exceeding a low threshold level $(0.6 \mu \mathrm{M})$, remains above it for a minimum time period and triggers LTD, and (3) a veto (V) detector which detects levels exceeding a mid-level threshold $(2 \mu \mathrm{M})$ and triggers a veto to the D response. A graphical schematic of the model neuron and its calcium detectors for spike timing dependent plasticity (STDP) is shown in Figure 1.

In this study we investigate how the pairing of an excitatory spike applied to the dendrite and an excitatory burst of action potentials applied to the soma both repeated every $300 \mathrm{~ms}$ (every theta cycle) affect the STDP in the dendrite in the presence and/or absence of high frequency inhibition applied to the dendrite.

\section{Experiments}

To investigate how the STDP profile is affected by the pairing of excitatory bursts and excitatory single spikes in the presence/absence of GABAergic inhibition,we designed the following experimental protocol: Excitatory burst of spikes with variable burst interspike interval and single spikes, which were repeatedly applied to the soma and dendrite, respectively, for $2 \mathrm{~s}(7$ times at about $3 \mathrm{~Hz}$ ) were paired in the absence and presence of an $100 \mathrm{~Hz}$ GABAergic inhibitory spike train applied between the excitatory pair interval $\Delta \tau$. Based on this protocol, we designed the following four physiological experiments (see figure 2), where the burst interspike interval, ISI, was allowed to vary:

$-\mathrm{ISI}=1.6 \mathrm{~ms}$

$-\mathrm{ISI}=5 \mathrm{~ms}$

- ISI $=10 \mathrm{~ms}$

$-\mathrm{ISI}=20 \mathrm{~ms}$

During all experimental paradigms, we varied the conductance of GABA inhibition and observed its effects on the amplitude of the dendritic $\mathrm{Ca}^{2+}$ spike and the STDP curve. These results are reported in the next section. 
A

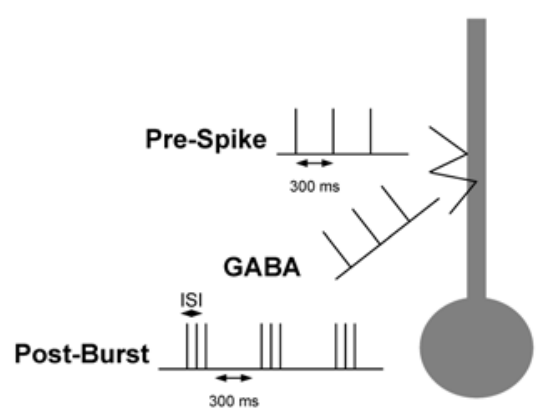

B

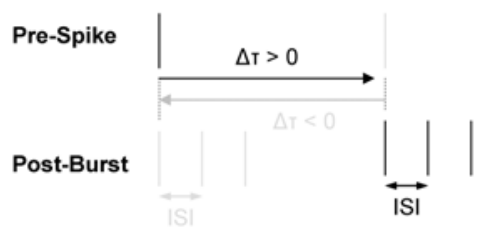

C

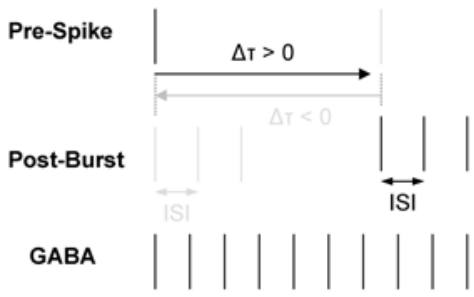

Fig. 2. (A) Model CA1 neuron with its three transient inputs to the soma and dendrite. Inputs: an excitatory burst to the soma, an excitatory spike to the dendrite and an inhibitory spike to the dendrite. Each input repeats every $300 \mathrm{~ms}$ for about 2 sec. (B) Pairing of a dendritic spike with a somatic burst in the absence of inhibition (GABA). (C) Pairing of a dendritic spike with a somatic burst in the presence of GABA inhibition.

\section{Results}

\subsection{Pairing of a Somatic Burst and a Dendritic Spike in the Absence of GABA Inhibition as a Function of Burst Interspike Interval}

In this section we investigate the effects of the burst ISI on the STDP profile in the absence of GABA inhibition. A presynaptic (dendritic) spike is paired with a postsynaptic (somatic) burst. The interstimulus interval $\Delta \tau$ is the interval between the presynaptic spike and the first spike ofthe postsynaptic burst (see Figure 2). The simulated STDP profile as a function of the interstimulus interval $\Delta \tau$ for different ISIs in the absence of GABA is depicted in Figure 3. A symmetric STDP profile centered at $+10 \mathrm{~ms}$ is evident for all ISIs. Interestingly the LTP value when the ISI is $5 \mathrm{~ms}$ and $10 \mathrm{~ms}$ is greater than when ISI is $1.6 \mathrm{~ms}$, but lower when ISI is $20 \mathrm{~ms}$.

In the preSpike10postBurst stimulation paradigm (see Figures 4A-L), where a presynaptic (dendritic) spike preceeds by $10 \mathrm{~ms}$ a postsynaptic (somatic) burst, calcium influx results from two sources: (1) through NMDA channels activated by the presynaptic spike, and (2) through VGCCs activated by the postsynaptic burst. This results in more than two calcium spikes depending on the ISI (see Figure $4 \mathrm{C}$ ). The first calcium spike, which is the result of the presynaptic spike 


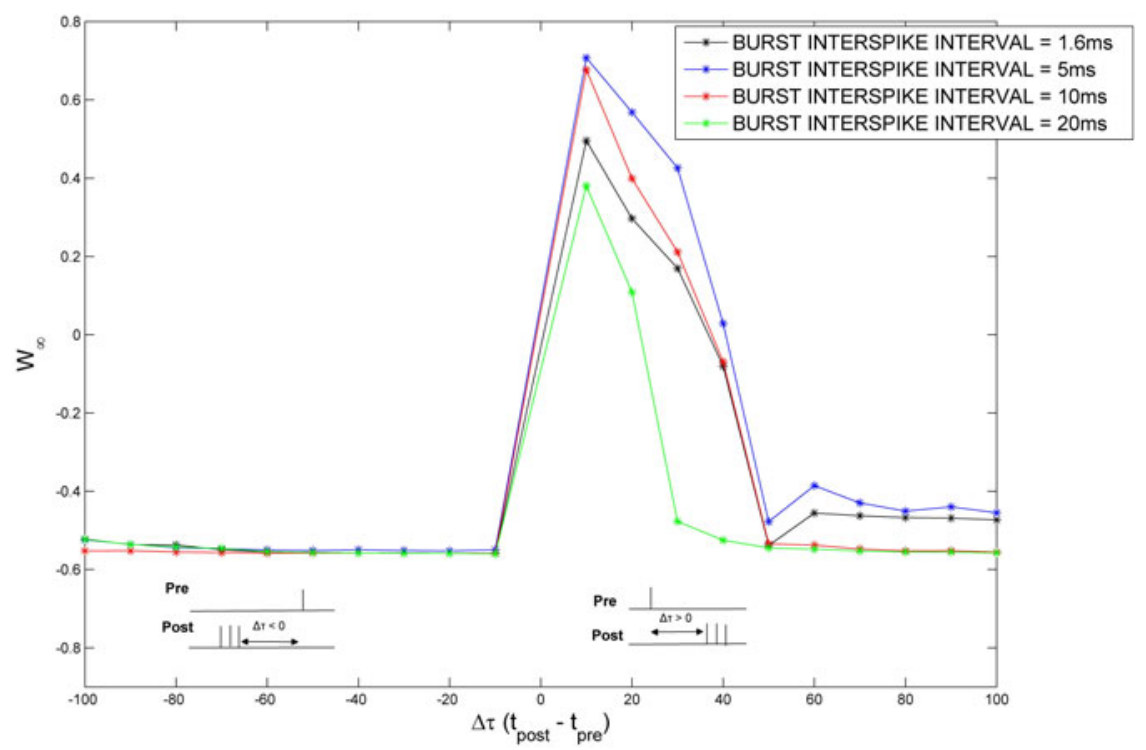

Fig. 3. Simulated asymmetric STDP profile as a function of burst interspike intervals in the absence of GABA inhibition. $\Delta \tau$ (tpost - tpre) is the interstimulus interval between the dendritic spike and the first spike of the somatic burst. $\Delta \tau$ ranges from -100 to 100 in increments of $10 \mathrm{~ms}$. (Inset-left) PostBurst - PreSpike scenario, where the dendritic spike follows by $\Delta \tau$ the somatic burst, comprised of three spikes. $\Delta \tau$ takes values from $-10 \mathrm{~ms}$ to $-100 \mathrm{~ms}$. The pairing repeats every $300 \mathrm{~ms}$. (Inset-right) PreSpike - PostBurst scenario, where a dendritic spike precedes the somatic burst by $\Delta \tau . \Delta \tau$ takes values from $+10 \mathrm{~ms}$ to $+100 \mathrm{~ms}$. The pairing repeats every $300 \mathrm{~ms}$.

activated NMDA channels, is always above $4 \mu \mathrm{M}$ regardless of the burst ISI. This high peak calcium spike on its own trigger all calcium detectors $(\mathrm{P}, \mathrm{D}$ and $\mathrm{V})$. Increases in $\mathrm{P}$ response assisted by the inhibition of the $\mathrm{D}$ response due to the activated $\mathrm{V}$ response will lead to the growth of $\mathrm{W}$ (see Figure 4D). In the case where burst ISI is either $5 \mathrm{~ms}, 10 \mathrm{~ms}$ or $20 \mathrm{~ms}$ additional calcium spikes will be generated (see blue, red and green traces in Figure 4C). When the ISI is $5 \mathrm{~ms}$ or $10 \mathrm{~ms}$, the second peak calcium values are above $4 \mu \mathrm{M}$ and the weight $(\mathrm{W})$ will be extra boosted (see Figure 4C). When the ISI is $20 \mathrm{~ms}$, the peak values of the third and fourth calcium peaks are all below the $4 \mu \mathrm{M}$ threshold, but still above the $2 \mu \mathrm{M}$ threshold. This means that the 3rd and 4th calcium spike will result from a reduced $\mathrm{P}$ response. But because they are above the $2 \mu \mathrm{M}$ threshold, the veto response will be activated, which will prevent the intermediate agents A and $\mathrm{B}$ from activating the depression (D) detector. Hence at ISI $=20 \mathrm{~ms}, \mathrm{~W}$ will plateau at lower value than when ISI is $1.6 \mathrm{~ms}, 5 \mathrm{~ms}$ and $10 \mathrm{~ms}$ (see Figure $4 \mathrm{D}-\mathrm{L})$. 
A

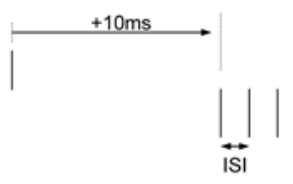

C

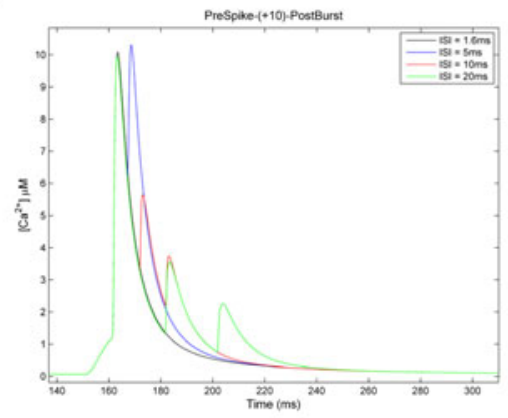

E

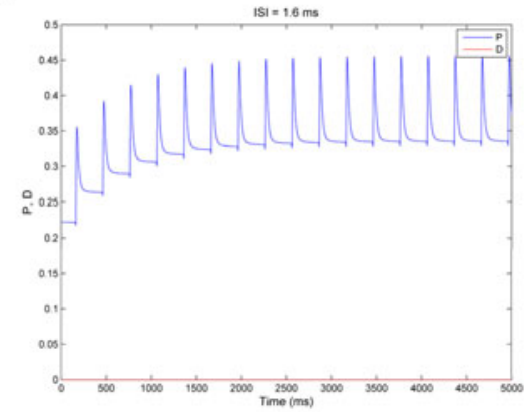

B

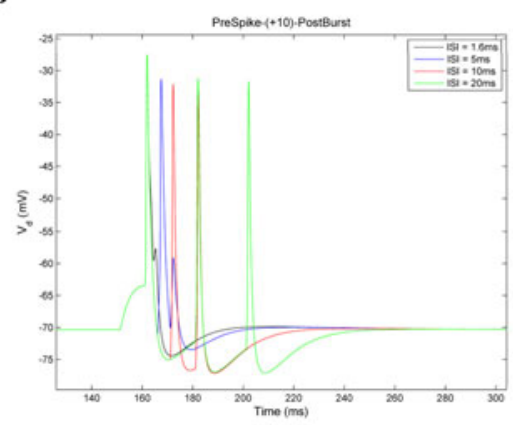

D

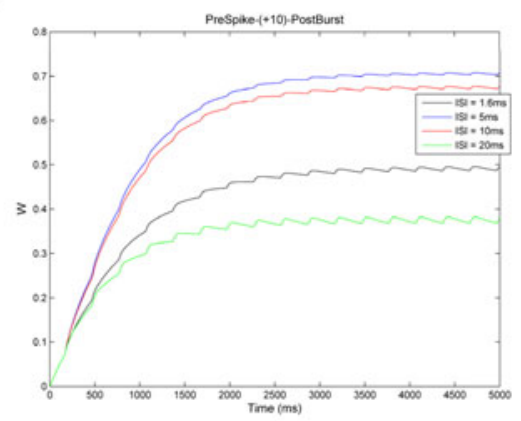

F

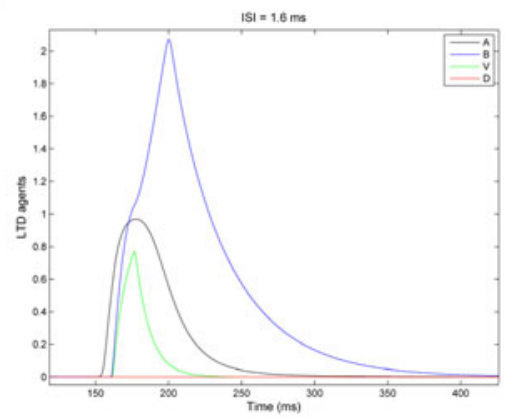

Fig. 4. (A) PreSpike-10-PostBurst stimulation paradigm used. The interstimulus interval between the dendritic spike (pre) and somatic burst (post) is set to $+10 \mathrm{~ms}$. (B-L) Direct comparison of $\mathrm{V}_{d},\left[\mathrm{Ca}^{2+}\right], \mathrm{W}, \mathrm{P}$ and $\mathrm{D}$, and LTD agents at various burst ISIs in the absence of GABA inhibition. 
G

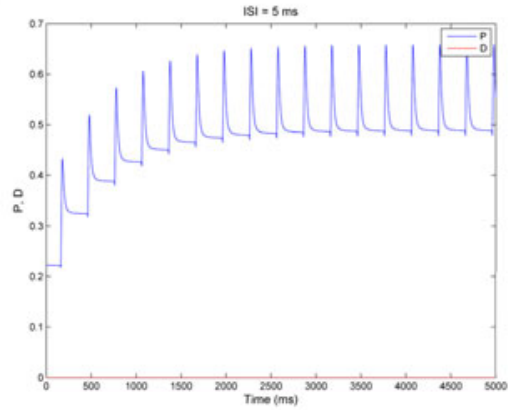

I

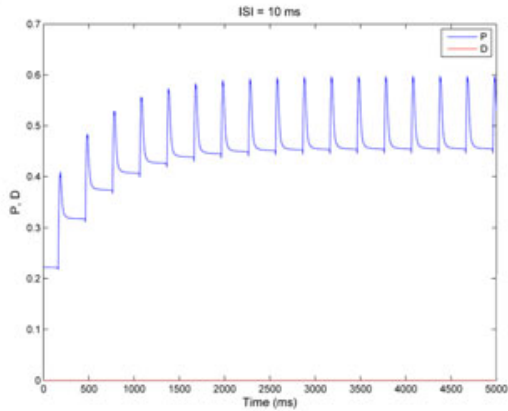

K

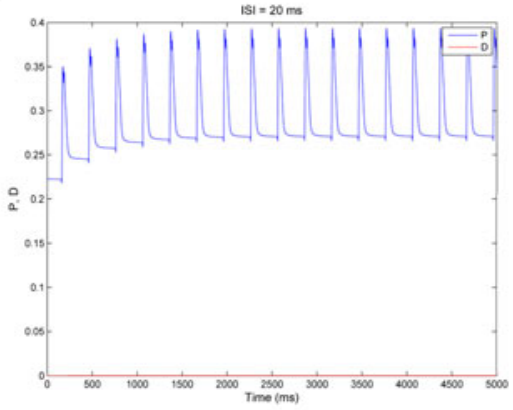

H

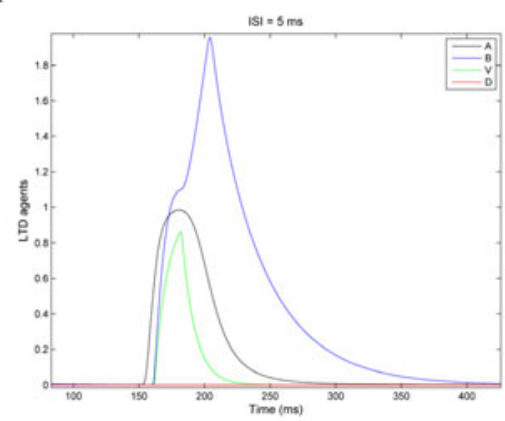

J

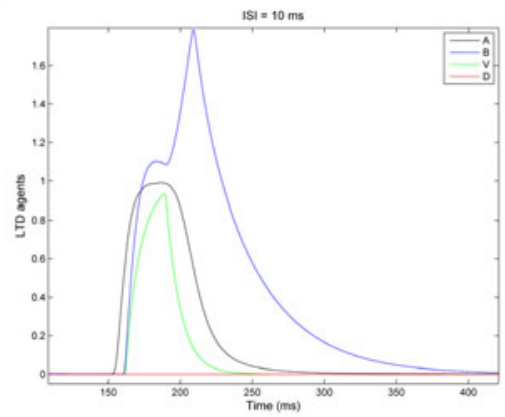

L

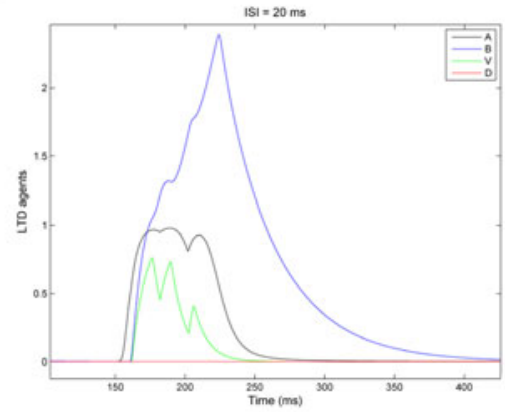

Fig. 4. (continued) 

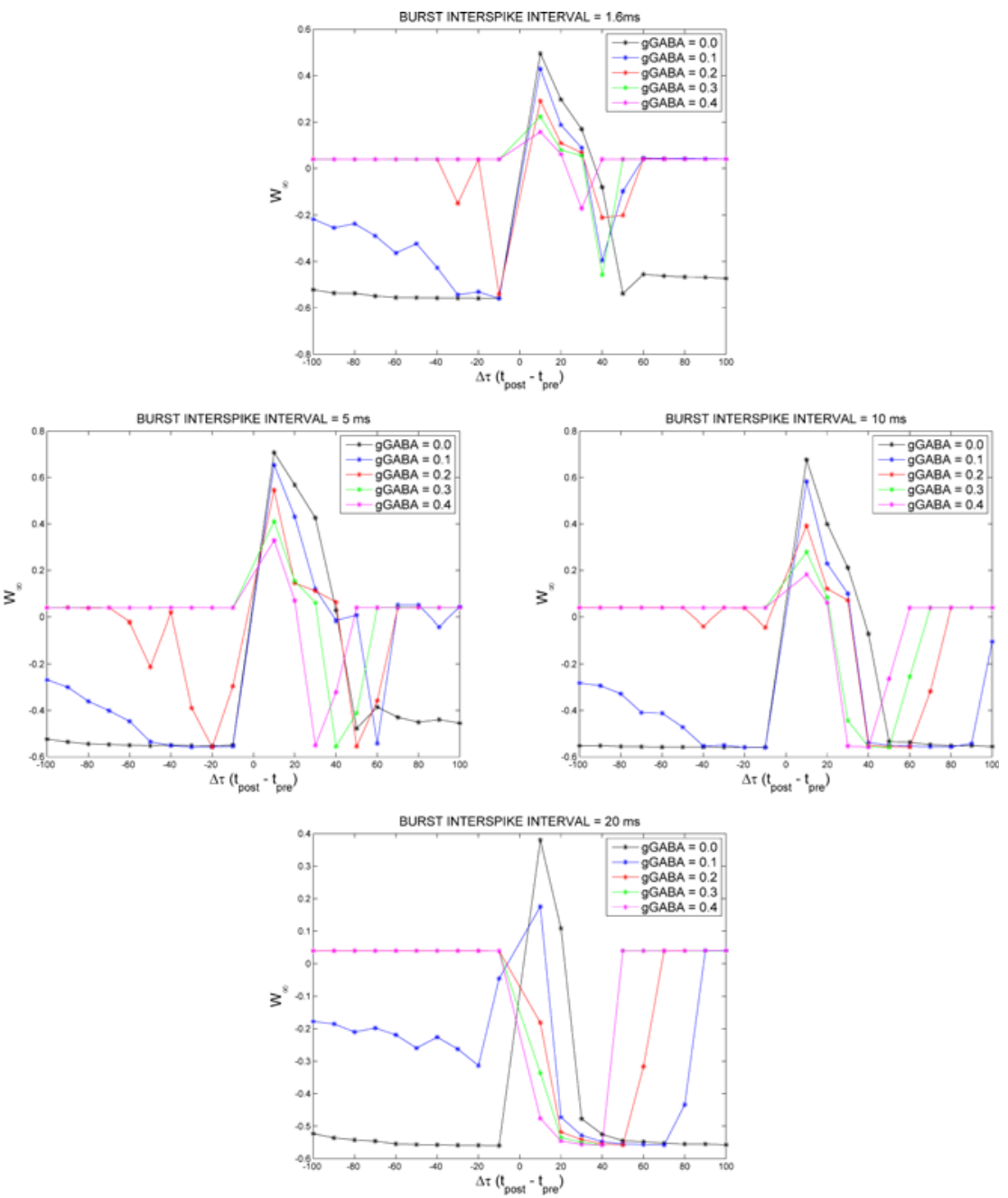

Fig. 5. STDP profiles from the pairing of a burst of spikes with variable burst interspike interval applied to the soma and a single spike applied to dendritic synapses in the absence and presence of a $100 \mathrm{~Hz}$ GABA spike train as a function of increasing GABA conductance. $\Delta \tau$ (tpost - tpre) ranges from -100 to 100 in increments of $10 \mathrm{~ms}$. (Top-left) Burst interspike interval is $1.6 \mathrm{~ms}$. (Top-right) Burst interspike interval is 5 ms. (Bottom-left) Burst interspike interval is $10 \mathrm{~ms}$. (Bottom-right) Burst interspike interval is $20 \mathrm{~ms}$. 


\subsection{Pairing of a Somatic Burst and a Dendritic Spike in the Presence of an $100 \mathrm{~Hz}$ GABA Spike Train as a Function of Increasing GABA Conductance}

Figure 5 is a composite figure of four graphs of $\mathrm{W}_{\infty}$ vs $\Delta \tau$ as a function of burst ISI and GABA conductance. The effect of GABA on the STDP profile is different for each ISI. When ISI is $1.6 \mathrm{~ms}, 5 \mathrm{~ms}$ and $10 \mathrm{~ms}$, the gaussian shaped STDP curve centered at $+10 \mathrm{~ms}$, is transforms to a Mexican hat one when gGABA $=$ $0.1-0.2 \mathrm{mS} / \mathrm{cm}^{2}$ to an asymmetric one at $\mathrm{g}_{G A B A}=0.3-0.4 \mathrm{mS} / \mathrm{cm}^{2}$. When ISI is $20 \mathrm{~ms}$, the gaussian shaped STDP curve switches to an a Mexican hat one with asymmetric LTD tails when gGABA is $0.1 \mathrm{mS} / \mathrm{cm}^{2}$ and to an inverted gaussian one when gGABA is greater than $0.1 \mathrm{mS} / \mathrm{cm}^{2}$ with the largest LTD value at $+30-40 \mathrm{~ms}$.

\section{Conclusion}

$\mathrm{A} \mathrm{Ca}^{2+}$ dynamics model of the CA1 pyramidal neuron with three calcium amplitude detectors was used to study the pairing effects of somatic AP bursts and dendritic single spikes on the spike timing dependent plasticity in the dendrite in the presence/absence of inhibition. In contrast to previous computational work [6] [7], 8], where an asymmetrical-to-symmetrical STDP curve is evident when somatic spikes are paired with dendritic bursts, in this study the STDP profile is symmetrical for all burst ISI and GABA conductance values. In the future, I intend to investigate whether this STDP symmetry is present when a dendritic burst is paired with a somatic one in the presence of different GABA gamma frequency sub-bands and conductance values.

Acknowledgement. This work was funded by the EPSRC project grant EP/ D04281X/1 and the NSF Science of Learning Center CELEST grant 0835976.

\section{References}

[1] Hebb, D.O.: The organization of behavior. John Wiley, New York (1949)

[2] Kampa, B.M., Letzkus, J.J., Stuart, G.J.: Requirement of dendritic calcium spikes for induction of spike-timing-dependent synaptic plasticity. J. Physiol. 574(1), 283-290 (2006)

[3] Letzkus, J.J., Kampa, B.M., Stuart, G.J.: Learning rules for spike timingdependent plasticity depend on dendritic synapse location. J. Neurosci. 26(41), 10420-10429 (2006)

[4] Allen, C., Stevens, C.F.: An evaluation of causes for unreliability of synaptic transmission. Proc. Natl. Acad. Sci. U.S.A. 91(22), 10380-10383 (1994)

[5] Bi, G.Q., Poo, M.M.: Synaptic modifications in cultured hippocampal neurons: dependence on spike timing, synaptic strength and postsynaptic cell type. J. Neurosci. 18, 10464-10472 (1998)

[6] Cutsuridis, V., Cobb, S., Graham, B.P.: A Ca ${ }^{2+}$ dynamics model of the STDP symmetry-to-asymmetry transition in the CA1 pyramidal cell of the hippocampus. In: Kůrková, V., Neruda, R., Koutník, J. (eds.) ICANN 2008,, Part II. LNCS, vol. 5164, pp. 627-635. Springer, Heidelberg (2008) 
[7] Cutsuridis, V., Cobb, S., Graham, B.P.: How Bursts Shape the STDP Curve in the Presence/Absence of GABA Inhibition. In: Alippi, C., et al. (eds.) ICANN 2009. LNCS, vol. 5768, pp. 229-238. Springer, Heidelberg (2009)

[8] Cutsuridis, V., Cobb, S., Graham, B.P.: Modelling the STDP Symmetry-toAsymmetry Transition in the Presence of GABAergic Inhibition. Neural Network World 19(5), 471-481

[9] Rubin, J.E., Gerkin, R.C., Bi, G.Q., Chow, C.C.: Calcium time course as signal for spike-timing-dependent plasticity. J. Neurophysiol. 93, 2600-2613 (2005)

[10] Aihara, T., Abiru, Y., Yamazaki, Y., Watanabe, H., Fukushima, Y., Tsukada, M.: The relation between spike-timing dependent plasticity and $\mathrm{Ca}^{2+}$ dynamics in the hippocampal CA1 network. Neuroscience 145(1), 80-87 (2007)

[11] Tsukada, M., Aihara, T., Kobayashi, Y., Shimazaki, H.: Spatial analysis of spiketiming-dependent LTP and LTD in the CA1 area of hippocampal slices using optical imaging. Hippocampus 15(1), 104-109 (2005)

[12] Nishiyama, M., Hong, K., Mikoshiba, K., Poo, M., Kato, K.: Calcium stores regulate the polarity and input specificity of synaptic modification. Nature 408, 584$589(2000)$

[13] Jarsky, T., Roxin, A., Kath, W.L., Spruston, N.: Conditional dendritic spike propagation following distal synaptic activation of hippocampal CA1 pyramidal neurons. Nat. Neurosci. 8(12), 1667-1676 (2005) 